\title{
Correction to: LinkGlide: A Wearable Haptic Display with Inverted Five-Bar Linkages for Delivering Multi-contact and Multi-modal Tactile Stimuli
}

Miguel Altamirano Cabrera and Dzmitry Tsetserukou

\author{
Correction to: \\ Chapter "LinkGlide: A Wearable Haptic Display \\ with Inverted Five-Bar Linkages for Delivering \\ Multi-contact and Multi-modal Tactile Stimuli" in: \\ H. Kajimoto et al. (Eds.): Haptic Interaction, LNEE 535, \\ https://doi.org/10.1007/978-981-13-3194-7_33
}

In the original version of the book, the given name and surname of the first author were identified incorrectly in chapter 33. The author's given name is Miguel and surname is Altamirano Cabrera.

The online version of the chapter has been corrected. 